\title{
ESTRUCTURA FISCAL Y DESCENTRALIZACIÓN EN MÉXICO: UN ANÁLISIS INSTITUCIONAL CON ÉNFASIS EN LA DISFUNCIONALIDAD EN EL USO DE RECURSOS DE LOS ESTADOS, 2016*
}

\section{Fernando Gaona Montiel ${ }^{1}$}

\section{Juan Mendoza Pérez}

\section{Resumen}

Este trabajo tiene el objetivo de analizar los factores institucionales que llevan a un problema de la estructura fiscal que, dada la falta de capacidad, la disfuncionalidad institucional y el tamaño del sector público, obligan a un financiamiento dependiente de las participaciones federales. Esto repercute en la debilidad de controles y la aplicación de la Ley, dada la dependencia de la Federación. En la evidencia estadística se halló que hay debilidades y disfuncionalidad en algunos estados frente a riesgos, y menores márgenes de maniobra, cuando se trata de evaluar el financiamiento de los gobiernos locales y las limitadas formas de recaudación local.

Palabras claves: Estado de derecho, disfuncionalidad, capacidad institucional y riesgos.

\begin{abstract}
This essay aims to analyze the institutional factors that lead to a problem of the fiscal structure, which given the lack of capacity and institutional dysfunction and the size of the public sector, require financial support of federal participations. That in turn impacts the weakness of controls

1 Profesor Asociado "D" tiempo completo, del Departamento de Economía de la UAM-I. Línea de investigación en Finanzas Públicas, Políticas Públicas y Macroeconomía.

2 Profesor Titular tiempo completo del Departamento de Economía de la UAM-I. Línea de investigación en Estudios Municipales y Descentralización Fiscal.

* Fecha de recepción: 28/11/2018. Fecha de aceptación: 21/01/2019.
\end{abstract}


and the application of the Law, for being dependent on the Federation. In the statistical evidence, it was found that there are weaknesses and dysfunction in some states, in the face of risks and the smaller margins of maneuver, when it comes to evaluating the financing of local governments and the limited forms of local collection.

Keywords: Rule of law, dysfunction, institutional capacity and risks.

\section{Introducción}

Hay un contraste entre la realidad de las finanzas públicas de los gobiernos locales y la estructura centralizada del sistema fiscal ${ }^{3}$, que refleja precariedad en el primero y excesos de recursos en el segundo. Hay una limitada capacidad de las instituciones, obligando a una dependencia del financiamiento de los ingresos federales. Las bondades de este sistema centralizado en los mecanismos de recaudación y de un sistema impositivo, si bien es cuestionable, ha sido extremadamente insuficiente en la recaudación descentralizada. Ello hace que simultáneamente se tenga un presupuesto restrictivo. Aún con las reformas no se observa una mejoría, debido a la existencia de un régimen fiscal centralizador en los hechos.

Los gobiernos federales y locales, por supuesto, son corresponsables de que operen adecuadamente las instituciones y que se atiendan los riesgos de las finanzas. Hay que reconocer el impacto y la valoración del papel de las instituciones, la informalidad, el estado de derecho y los derechos de propiedad. La eficiencia en esto radica en el cómo se vea su capacidad institucional de gobernar y si son aceptables y legítimas sus acciones. Las políticas públicas, en su diseño e implementación, pueden detectar problemas en el proceso de recaudación y gasto, lo que impide la realización de una descentralización fiscal (Cepal, 1993: $8)^{3} 4$ más efectiva.

3 La autoridad fiscal aplica impuestos y gastos, con base en una división entre gobierno central y los gobiernos estatales y municipales.

4 Se entiende como un proceso de transferencias de competencias y recursos desde la administración central hacia la administración estatal o municipal. 
Este trabajo tiene el propósito de analizar los factores institucionales que están implícitos dentro del problema de la estructura fiscal, que se detecta una falta de capacidad y disfuncionalidad institucional y lleva a un financiamiento dependiente de las participaciones federales. Existe un problema de capacidad institucional y de poca sostenibilidad de la estructura fiscal, debido a la alta dependencia de los recursos de la Federación. De ahí que la descentralización viene atada a la propia centralización de recursos federales, que emana del propio sistema. Es necesario verificar las bases mínimas y criterios que tienen que seguirse en torno a la descentralización fiscal.

Las reglas burocráticas que rigen los procesos, procedimientos y las acciones rutinarias siguen siendo favorables y ventajosas al gobierno federal. Aunque en este tema no se realice un cabal compendio del proceso si conlleva un asunto de gestión, organización y de insuficiencias en el desarrollo institucional del sistema. Esto merece una atención técnica en un sentido más amplio cuyas reglas y organizaciones sean competentes en los tres distintos niveles de gobiernos: municipal, estatal y federal.

Ello impone límites tanto a nivel federal y en los gobiernos locales, dada las incapacidades de las instituciones locales, que obligan a un financiamiento dependiente de las participaciones federales (Alor \& Valenzuela, 2011 $)^{5}$. Es posible hacer un recuento que hace evidente la debilidad y disfuncionalidad en la capacidad institucional del sistema. A pesar de ser difícil probar la existencia de disfuncionalidades del sistema frente a riesgos se imponen menores márgenes de maniobra en el financiamiento de los gobiernos locales, como unas formas de recaudación local demasiado dependientes de la potestad federal.

El gobierno local necesita destrabar reglas y mecanismos para recaudar impuestos y otros ingresos, a efecto de apoyar sus propias políticas públicas. Se busca no sólo valorar mecanismos de recaudación dentro del sistema tributario, sino hacer una reflexión sobre la pertinencia de

5 Es un gasto descentralizado que hace referencia a los ramos 28 y 33, principalmente, que representan el manejo de más de cinco fondos, que son más del $90 \%$ del gasto total descentralizado, aprobado para un ejercicio fiscal. 
continuar dependiendo de la federación y del papel que le corresponde en un sistema fiscal centralizador. Esta situación se verifica en las finanzas públicas de los gobiernos locales y existe una limitada participación en los ingresos federales. Ello hace de éste un sistema disfuncional en su capacidad institucional, que se agrava y se vuelve fuente de riesgos e inestabilidad conforme pasa el tiempo.

\section{Análisis del problema del sistema fiscal federal y local: enfoque institucional}

El criterio institucional (Knack y Keefer, 1995) del sistema y las formas de descentralización se vuelve útil ${ }^{6}$ como apoyo para evaluar los procesos sustantivos del gobierno; toda vez que implica la revisión de concentrar los esfuerzos en la distribución territorial de recursos (oficinas, equipos y personal) y la verificación de las funciones de vigilar y regular, con reglas y procedimientos institucionales las formas de recaudación de ingresos fiscales y de distribución del gasto local. Cabe destacar, que una teoría institucional se halla en Weber (2014), quien toca a las organizaciones como estructuras políticas y legales, con reglas para determinar el tipo de gobernanza. Para Meléndez y Maldonado-Veloza (2014), se hallan factores positivos y negativos que influyen en la eficiencia de las instituciones, los cuales condicionan el impacto y la posible gobernabilidad en los Estados.

Entre el Estado y la sociedad se construye una estructura fiscal, pero lo deseable es que sea eficiente; no obstante, no es fácil de evaluar si lo es o no. Es factible ver la creación de organizaciones formales en todos los ámbitos de la vida social (Meyer y Scott, 1983; Scott y Meyer, 1994) cuando se atiende la institucionalización de procesos homogéneos, que tiene lugar en la manera en que las organizaciones tienden a ser semejantes en sus características estructurales y funcionales. En

6 La importancia de factores institucionales remite a los trabajos de Thorstein Veblen, Wesley Mitchell y John Commons, que en la actualidad son retomados por autores como Knack y Keefer (1995), quienes aseguran que el ambiente y la calidad de instituciones influye en la actividad económica de algunos países. 
Powell y DiMaggio (1991) analizan el efecto de la homogeneidad de los procesos dentro de las instituciones.

Las reglas y las organizaciones burocráticas, según Weber (2014, 2011), si influyen en formas organizaciones más racionales y técnicamente eficientes. Además, en Weber la burocratización inicialmente promueve la competencia y la eficacia de las organizaciones y, posteriormente, considera que el resultado de dicha estructura burocrática se convierte en procesos homogéneos. Para Maguire y Hardy (2009), la institucionalización es un proceso que no se perpetúa a lo largo del tiempo por el abandono de ciertas prácticas sociales. En efecto, estos autores señalan que los agentes promueven y caen en un conflicto, bajo riesgo de confrontación, por los mecanismos coercitivos, normativos y del conocimiento cultural, lo que no ayuda a los procesos.

Según datos de la Secretaría de Hacienda y Crédito Público (SHCP), las administraciones públicas locales llegaron a disponer de recursos por 1.6 billones de pesos en 2016, casi el 35\% del gasto total de la federación. Se le conoce como gasto federalizado ${ }^{7}$ a través del acceso a recursos públicos de las participaciones y aportaciones federales, además de otros ramos de gasto. En Rosen (2002), no es posible ni es válida la crítica del gobierno federal a la administración y el manejo del gasto de los gobiernos locales. Cuando interviene el gobierno federal ${ }^{8}$ toma decisiones a nivel local, se producen distorsiones y se generan ineficiencias del presupuesto. Los trabajos de Wallace Oates (1969) y otros autores, establecen la evidencia de que cualquier bien público que se produzca en los gobiernos locales será más eficiente, en sus respectivas jurisdicciones, frente a la posible provisión de un gobierno central (Oates, 1969)9 .

7 Incluye las aportaciones federales (ramo 33), participaciones federales (ramo 28), convenios de descentralización, protección en salud (seguro popular), previsiones en educación (ramo 25) y gasto del ramo 23.

8 Hace referencia al sector público con varios niveles de decisiones, una posición central o descentralizada, dependiendo de cada área de jurisdicción (Oates, 1972).

9 Con base en el "teorema de descentralización", formulada por Wallace Oates (1969). 
En los estudios de la incidencia de impuestos a la propiedad, existen también casos en que este impuesto es posible dar buenos resultados, así como en los programas de gastos en los gobiernos locales. La configuración de un orden fiscal por entidades no ha sido sobre las bases de una institucionalización y la creación de un estado de derecho, o de suscribir las disposiciones jurídicas, que les permita a los gobiernos una mayor legitimidad para ser una autoridad fiscal que le dé fortaleza. La constitución se sienta sobre un federalismo y un marco jurídico que despliega todos los esfuerzos por una mayor soberanía local.

Al contrario, pareciera que hay más razones para pensar en acciones que dan lugar a una "desinstitucionalización" del sistema fiscal, por la alta rotación del cuerpo profesional dedicado a la recaudación y la desaparición de áreas que intentan aumentar la capacidad institucional en las entidades, sin lograrlo a cabalidad. Subsiste la contradicción por parte de los gobiernos locales, entre dar cauce a una estructura institucional más fuerte que aplica las leyes o la postura política de rehacer las instituciones para que las regulaciones del Estado no impidan las libertades en los negocios y se afiancen las actividades productivas. No obstante que no haya la suficiente recaudación que impulse con recursos propios la oferta de servicios públicos mínimos, a pesar de ser dependientes de los recursos federales.

Hay factores de tipo institucional que impiden la construcción de un sistema fiscal sólido y fuerte frente a una recaudación que posee mecanismos centralizadores, que involucra las deficiencias en la capacidad institucional del sistema fiscal ${ }^{10}$. Para Béjar (2010), existe un federalismo de corte pluralista en los hechos orientado hacia un reparto del poder político que, si bien se funda en el marco constitucional, también se mueve en función de los reclamos sociales. La política fiscal se encuentra en una encrucijada por ahora, tiene que ver con un sistema de recaudación federal, que limita y ahoga las finanzas de los gobiernos locales. Se cuestiona realmente si hay capacidad de pago para enfren-

10 Como sistema nos vamos a referir al Sistema de Administración Tributaria (SAT) y todas las instituciones públicas que tienen relación con el proceso de tributación-gasto en los tres órdenes de gobierno. 
tar las obligaciones contraídas por la deuda pública en los gobiernos locales y la insuficiencia de sus propios ingresos fiscales.

La teoría económica tradicional omite la presencia de las instituciones (Eggertsson, 1990) cuando se trata de actos de mercado y que el gobierno no puede dejar de lado por afectar el comportamiento de los individuos. Obviamente, involucra las conductas de las empresas en razón de la existencia de reglas y organizaciones, que de no apegarse a ellas motivan la afectación del crecimiento de la economía. Sin duda, los gobiernos locales tienen que adecuar esas reglas y el sistema organizacional, a las condiciones de la región, a fin de brindar certidumbre y patrones de conducta apropiados para resguardar los derechos de propiedad y el cumplimiento de contratos.

Una concepción tradicional de la macroeconomía no ve la importancia de las instituciones, en las cuales confluyen la actuación de individuos, las acciones de gobierno, intereses, contratos y relaciones de propiedad (Williamson, 1989). El nuevo enfoque institucional (Meléndez y Maldonado-Veloza, 2014) ${ }^{11}$, a diferencia del institucionalismo de Thorstein Veblen, permite integrar la teoría económica al desarrollo de una economía; esta vez puede lograr la confluencia del papel de los gobiernos estatales y la evolución institucional en búsqueda de un modo eficiente de asignación de recursos. Es decir, se trata de ver la pertinencia de las estructuras administrativas, en conjunción con la gobernabilidad y las políticas públicas que llevan a una mejoría de las finanzas públicas locales. Ello atiende a buscar una distribución eficiente de los recursos públicos. En Dani Rodrik (2000) y Graham, Grindle, Lora y Seddon (1999), afirman que no se trata de ver sí son importantes o no las instituciones, sino del papel que pueden jugar en la reforma de Estado y que permitan instaurar la gobernabilidad, dejando paso a una posible descentralización fiscal de mayor cobertura.

11 Las instituciones son las que establecen las reglas del juego y determinan "el qué, cómo, cuándo y dónde"; mientras que las organizaciones describen a los jugadores, como entes participantes. El Estado y el sistema de justicia debe vigilar el cumplimiento de las normas (Meléndez y Maldonado-Veloza, 2014: 63). 
La racionalidad toma relevancia en las decisiones óptimas de los individuos, pero queda sujeta a la información disponible. El sistema fiscal y el proceso de descentralización necesita adaptación a las condiciones distintas de cada entidad, de lo contrario se generan disfuncionalidades. Si bien los individuos actúan y forman parte de las instituciones, éstas pueden ser diferentes por entidad, dadas las condiciones económicas; pero su conocimiento es dependiente del uso racional y sus actos del tipo de actividades económicas. En Williamson (1989: 22), se advierte que Hebert Simon $(1961,1972)$ encontró que el comportamiento humano es de "intención racional", pero sólo limitadamente, debido la información disponible y la existencia de una realidad económica que difiere de una entidad a otra.

Las capacidades institucionales de los gobiernos locales, a veces limitadas e insuficientes, hacen posible el surgimiento de riesgos y conflictos que pueden provocar una situación no estable en la economía. No existe mucho margen para modificar la estructura tributaria de los gobiernos locales, las características son desalentadoras, como lo expresan Pierre-Richard \& Montiel (2000) y Goode (1984), la administración tributaria es débil e insuficiente. Desde un punto de vista de la estructura organizativa, sino se toma en cuenta, las expectativas de una reforma fiscal de mayor profundidad serían entonces de facto una capacidad institucional ineficiente.

El realismo de una economía es más amplio, no es sólo de mercados, sino de instituciones. La situación de las finanzas públicas, sin duda, se localiza en un ámbito de normas e instituciones públicas que en mucho de los casos tiene relación con la capacidad institucional de llevar a cabo un federalismo fiscal. Analizar la solvencia y la capacidad financiera de los gobiernos locales, que se vislumbra en las finanzas públicas, no es más que un medio para advertir los riesgos de continuar bajo la dependencia de un financiamiento de los ingresos federales y de deuda pública. 


\section{Existencia de disfuncionalidades en la capacidad institucional del sistema fiscal}

La competencia fiscal en materia de recaudación y gasto implica un proceso de transferencia de ciertas facultades a las entidades locales, o bien, estados sub-nacionales. El transferir recursos y competencias conlleva, sin desearlo, generar disfuncionalidades ${ }^{12}$ en las instituciones y diversas repercusiones fiscales para el gobierno. Sin duda, en el país existe un alto grado de centralización que está generando grandes disfuncionalidades como obstáculos para el desarrollo y pocos espacios de gobernabilidad (Cabrero, 1996). En realidad, es un proceso en que se dan cambios institucionales, económicos y de política, que no son neutrales en sus resultados, pero sí hay costos adicionales en las finanzas (Cepal,1993: 27). Asimismo, se da en un contexto de una división territorial en unidades inferiores de gobiernos locales, que vienen a depender de un gobierno central.

La disfuncionalidad aparece en las instituciones cuando no hay un funcionamiento adecuado y los resultados no son los deseables. La no funcionalidad en el sistema fiscal, en los tres órdenes de gobierno, se da al menos por tres motivos:

a) De tipo administrativo. No hay capacidad administrativa ni personal para resolver la precariedad de los ingresos fiscales, además de mejorar el sistema de cobros y plazos en los impuestos.

b) De tipo financiero. Cuando existe principalmente una fuerte dependencia de las administraciones locales ante los recursos públicos que distribuye la federación.

c) De tipo legal. Las facultades de los gobiernos locales en cuanto a las formas de aplicar los gravámenes está supeditada en la Ley, el apego a ésta depende del grado de autonomía frente a la federación.

12 Con las reformas del Artículo 115 de la Constitución, en 1999, se buscó darle mayores competencias y cierta autonomía a los Estados y municipios, recayendo en los Congresos locales, el determinar los impuestos, los plazos de cobro y bases tributarias (Cabrero, 1996). 
En el ámbito de la administración de los gobiernos locales, se cuentan con demasiadas funciones y responsabilidades, pero la mayoría no dispone de la capacidad ni de la infraestructura administrativa suficiente, lo que lleva a un fenómeno de disfuncionalidad en las instituciones $\mathrm{y}$ de incapacidad en el sistema fiscal.

El tema de federalismo fiscal y la descentralización (Rodden, 2005) remite necesariamente a un asunto de disfuncionalidad en la capacidad institucional, que de no corregirse despliegan problemas en la relación entre gobierno federal y los estados ${ }^{13}$, dependiendo de los recursos provenientes de la federación. Sin embargo, se debe reconocer la existencia de escasos incentivos que motiven a elevar los ingresos y las fuentes de recursos propios. En Jonathan Rodden (2003), a medida que el gobierno federal aumenta las transferencias a los gobiernos locales, éstos no hacen lo suficiente para disminuir su gasto y sí agotan todos los recursos. No existe una receta o esquema que apoye en una mayor eficiencia del gasto y los ingresos (Ramírez, 2012) ${ }^{14}$.

Existe un sistema de transferencias y participaciones hacia los gobiernos locales, pero no hay un mecanismo para reconocer la eficiencia en el gasto ni en las formas de recaudación; específicamente, al constituir reglas y criterios apropiados para la existencia de incentivos que generen condiciones para que las entidades establezcan más impuestos recaudatorios en el ámbito de su competencia.

El financiamiento de los gobiernos estatales y municipales obedece al tipo de prácticas de gasto y recaudatorias que efectúan los gobiernos, pero no hay suficientes ingresos propios por impuestos (impuestos sobre ingresos, la nómina y el patrimonio, etc.) ni del tipo no tributarios, como los derechos, productos, aprovechamientos y contribuciones de

13 En Rodden (2005), la estructura federal y la descentralización no llega a ser lo mismo, subsiste el problema de la autonomía del gobierno central, limitada por reglas constitucionales o restricciones informales.

14 Los gobiernos estatales y municipales se concentran más en gravar los activos fijos (herencias en propiedades, bienes inmuebles), como los impuestos predial y el patrimonio (Ramírez, 2012). 
mejoras. Tan es así, que la elevación del gasto en los gobiernos locales, depende más de los recursos públicos transferidos por la Federación.

Resulta evidente, que los componentes de los ingresos fiscales presentan enormes diferencias en las fuentes de sus recursos. De acuerdo a datos del INEGI, durante 2016, se observó que el conjunto de las entidades tuvo un ingreso fiscal promedio del $8.8 \%$ en las entidades que provienen de impuestos ${ }^{15} \mathrm{y}$ de una recaudación no tributaria ${ }^{16}$ (Cuadro 1), por debajo de sus ingresos fiscales totales de cada entidad; lo que significa su alta dependencia de los recursos federales. Entre las entidades que superaron este promedio se encuentran: Coahuila, Chihuahua, Guanajuato, Estado de México, Nuevo León, Querétaro y Quintana Roo. Al contrario, las entidades que tuvieron menores ingresos por estas fuentes fueron: Guerrero, Tlaxcala y Zacatecas, entre otras.

\section{Cuadro 1. Estructura de los ingresos fiscales por entidades, 2016 $^{\mathrm{a} /}$ (Miles millones pesos corrientes, \%)}

\begin{tabular}{|c|c|c|c|c|c|c|}
\hline \multicolumn{2}{|c|}{} & $\begin{array}{c}\text { Total } \\
\text { Ingresos } \\
\text { Entidad }\end{array}$ & Impuestos & $\begin{array}{c}\text { Recauda- } \\
\text { ción no } \\
\text { tributaria }\end{array}$ & $\begin{array}{c}\text { Suma de } \\
\text { Recauda- } \\
\text { ción }\end{array}$ & $\begin{array}{c}\text { Recauda- } \\
\text { ción al total } \\
\text { Ingresos } \\
\text { Fiscales }\end{array}$ \\
\cline { 2 - 7 } & A & B & C & B + C & B + C / A (\%) \\
\hline $\mathbf{0 1}$ & $\begin{array}{c}\text { Aguasca- } \\
\text { lientes }\end{array}$ & $24,431.9$ & 926.4 & $1,163.6$ & $2,090.0$ & 8.6 \\
\hline $\mathbf{0 2}$ & $\begin{array}{c}\text { Baja } \\
\text { California }\end{array}$ & $50,306.6$ & $3,003.3$ & $1,347.0$ & $4,350.3$ & 8.6 \\
\hline $\mathbf{0 3}$ & $\begin{array}{c}\text { Baja Cali- } \\
\text { fornia Sur }\end{array}$ & $18,423.1$ & 763.0 & 554.8 & $1,317.8$ & 7.2 \\
\hline $\mathbf{0 4}$ & Campeche & $21,475.8$ & $1,544.7$ & 564.1 & $2,108.7$ & 9.8 \\
\hline $\mathbf{0 5}$ & $\begin{array}{c}\text { Coahuila de } \\
\text { Zaragoza }\end{array}$ & $46,495.0$ & $3,362.8$ & $3,676.8$ & $7,039.6$ & 15.1 \\
\hline $\mathbf{0 6}$ & Colima & $16,146.7$ & 647.7 & 487.5 & $1,135.2$ & 7.0 \\
\hline $\mathbf{0 7}$ & Chiapas & $90,094.4$ & $1,471.2$ & $1,124.9$ & $2,526.4$ & 7.8 \\
\hline
\end{tabular}

15 Entre los gravámenes estatales están los impuestos al predial, la nómina (remuneraciones al trabajo), el ejercicio de la profesión y honorarios, hospedaje y la enajenación de vehículos usados.

16 Contribuciones de mejoras, derechos (uso y aprovechamiento de bienes de dominio público), productos (explotación de bienes patrimoniales y productos financieros), aprovechamientos (ingresos por recargos, intereses moratorios, multas, etc.). 


\begin{tabular}{|c|c|c|c|c|c|c|}
\hline 08 & Chihuahua & $60,965.1$ & $3,989.0$ & $4,782.3$ & $8,771.3$ & 14.4 \\
\hline 09 & Durango & $32,524.2$ & $1,401.5$ & $1,124.9$ & $2,526.4$ & 7.8 \\
\hline 10 & Guanajuato & $86,056.6$ & $2,952.2$ & $10,312.6$ & $13,264.8$ & 15.4 \\
\hline 11 & Guerrero & $61,819.2$ & $1,194.2$ & 403.7 & $1,597.9$ & 2.6 \\
\hline 12 & Hidalgo & $46,099.0$ & $1,394.9$ & 871.6 & $2,266.4$ & 4.9 \\
\hline 13 & Jalisco & $103,116.2$ & $4,335.5$ & $4,199.2$ & $8,534.6$ & 8.3 \\
\hline 14 & México & $275,773.7$ & $16,991.6$ & $32,110.5$ & $49,102.0$ & 17.8 \\
\hline 15 & $\begin{array}{l}\text { Michoacan } \\
\text { de Ocampo }\end{array}$ & $68,479.3$ & $1,061.6$ & $2,351.2$ & $3,412.8$ & 5.0 \\
\hline 16 & Morelos & $30,371.8$ & 730.1 & $1,002.5$ & $1,732.6$ & 5.7 \\
\hline 17 & Nayarit & $21,889.8$ & 773.2 & 354.7 & $1,127.9$ & 5.2 \\
\hline 18 & Nuevo León & $94,225.9$ & $9,024.9$ & $7,163.3$ & $16,188.2$ & 17.2 \\
\hline 19 & Oaxaca & $70,535.3$ & $1,172.0$ & $2,389.2$ & $3,561.1$ & 5.0 \\
\hline 20 & Puebla & $91,006.2$ & $4,091.3$ & $3,262.5$ & $7,353.8$ & 8.1 \\
\hline 21 & Querétaro & $34,073.3$ & $2,733.0$ & $2,234.9$ & $4,967.9$ & \\
\hline 22 & $\begin{array}{c}\text { Quintana } \\
\text { Roo }\end{array}$ & $28,443.5$ & $2,585.5$ & $1,981.1$ & $4,566.6$ & 16.1 \\
\hline 23 & $\begin{array}{c}\text { San Luis } \\
\text { Potosí }\end{array}$ & $45,899.7$ & $1,410.4$ & $1,830.1$ & $3,240.5$ & 7.1 \\
\hline 24 & Sinaloa & $49,226.3$ & $1,815.1$ & $2,497.9$ & $4,313.0$ & 8.8 \\
\hline 25 & Sonora & $63,927.6$ & $2,482.4$ & $1,974.5$ & $4,456.9$ & 7.0 \\
\hline 26 & Tabasco & $48,999.2$ & $1,563.6$ & $1,490.0$ & $3,053.6$ & 6.2 \\
\hline 27 & Tamaulipas & $54,481.1$ & $2,287.6$ & $2,896.2$ & $5,183.7$ & 9.5 \\
\hline 28 & Tlaxcala & $22,005.4$ & 357.4 & 501.8 & 859.2 & 3.9 \\
\hline 29 & $\begin{array}{l}\text { Veracruz de } \\
\text { Ignacio de } \\
\text { la Llave }\end{array}$ & $101,808.2$ & $3,905.0$ & $3,039.9$ & $6,944.9$ & 6.8 \\
\hline 30 & Yucatán & $35,643.3$ & $1,730.4$ & $1,493.0$ & $3,223.4$ & 9.0 \\
\hline 31 & Zacatecas & $34,484.9$ & 424.2 & $1,118.7$ & $1,542.9$ & 4.5 \\
\hline \multicolumn{2}{|c|}{ Total nacional } & $1,829,228.6$ & $82,125.7$ & $102,298.0$ & $184,423.7$ & 8.8 \\
\hline
\end{tabular}

Fuente: Elaboración propia con datos del INEGI, Finanzas públicas estatales y municipales.

a/ No incluye a la Ciudad de México.

1 Impuestos sobre los ingresos, sobre nómina, sobre el patrimonio, sobre tenencia o uso de vehículo, etc.

2 Contribuciones de mejoras, derechos, productos y aprovechamientos. En el caso del Estado de méxico se incluyen cuotas y apostaciones a la seguridad social. 
Estas diferencias en la recaudación de impuestos y no impositiva hace que los financiamientos de los gobiernos tengan que venir de la Federación. Pero, también se advierte que existe una variedad de esfuerzos fiscales que no corresponden a la participación de los fondos federales; ya sea de las partidas como las aportaciones del Ramo 33, los recursos federales reasignados, ingresos de empresas estatales, empréstitos. Además, se hallan las participaciones federales, los fondos participables y el fondo general de participaciones.

Esto se ha replicado a los gobiernos locales, puesto que el desarrollo desigual de las economías trajo consigo una disparidad en las capacidades institucionales del sistema, aunado a un esfuerzo fiscal que no es parejo al momento de la recaudación en los gobiernos estatales y municipales. Hay estados que dependen de la tributación sobre inmuebles y los servicios públicos, pero no existe la responsabilidad ni el interés por elevar la recaudación con base en ingresos propios y, así, emprender programas sociales. Por la difícil situación de las finanzas locales, resulta inaplazable la ejecución de la descentralización fiscal y la de transferir funciones para la fortaleza de estos gobiernos.

Entre los factores que contravienen la tendencia a fortalecer la capacidad institucional, y que ocasiona una disfuncionalidad perniciosa, es la persistencia en la minimización de los problemas alrededor del fenómeno conocido, como selección adversa y riesgo moral, que es una obligación de gobiernos locales ${ }^{17}$ de cuidar su prestigio y calidad crediticia (García, 2014). Eso no es todo, el problema es el análisis de la deuda municipal y el cómo la SHCP hace los registros contables, pero ello no da cuenta de toda la deuda contratada y la información de su destino y el vencimiento (García, 2014) ${ }^{18}$.

17 La calificación crediticia se otorga por una institución evaluadora y los bancos asignan un riesgo crediticio al gobierno municipal. Es una obligación de los municipios, no sólo de las entidades federativas (García, 2014: 131).

18 Por recomendación de la Auditoría Superior de la Federación, a partir del ejercicio fiscal 2014, el Registro de Obligaciones y Empréstitos de Entidades Federativas y Municipios de la Secretaría de Hacienda permite obtener más información acerca de las características de contratación (García, 2014). 
Otra situación ocurre con las transferencias del gobierno central a los gobiernos locales, pues ocasionan que haya una percepción de que los gobiernos sean benevolentes y provean servicios y bienes públicos, más allá del óptimo social y de los recursos tributarios disponibles (Sour y Girón, 2008). Además, se encuentra que hay una serie de problemas en la posibilidad de realizar una descentralización, pues esto no sólo involucra lo fiscal sino que implica atender aspectos institucionales y legales, de infraestructura técnica y gestión de los servidores públicos (Cabrero y Carrera, 2001).

\subsection{La estructura administrativa y gestión estatal de las finanzas}

La gestión y el conocimiento de los recursos públicos, dentro de las áreas de contabilidad y del presupuestal en los gobiernos de los estados, no es una estructura que se aprecie eficiente y ágil, sino que está inmersa en diversos mecanismos de control. Las funciones atienden asuntos de ingresos de gasto y de operaciones en torno a la deuda estatal. Pocas secretarías de finanzas llevan una programación por programas, que merezcan un apoyo más amplio del tipo tecnológico e informático en el seguimiento y control del presupuesto. Sin duda, el interés por llevar a cabo los trabajos de capacitación, por áreas presupuestales, está dependiendo de si se desea mejorar el desempeño y la capacidad institucional en el desempeño financiero, el cual muchas veces está resultando con claras deficiencias y problemas de responsabilidad administrativa.

No hay duda que las mayores presiones políticas y de rendición de cuentas se localizan en los gobiernos locales; máxime si operan gobiernos participativos, cabildos abiertos a la ciudadanía, comités de colonos, contralorías sociales y la formación de fideicomisos (Cabrero, 2004: 763). La información tiene que hacerse pública, abierta al escrutinio de la ciudadanía, lo que implican la construcción de mecanismos de difusión y transparencia, con el uso de nuevas tecnologías informáticas $\mathrm{y}$ de redes.

De acuerdo al INEGI, del Censo Nacional de Gobiernos Municipales y Delegacionales, en el año 2016 la mayor parte de la gestión en trá- 
mites y servicios se concentran en un $50.1 \%$ en los pagos del impuesto predial, mientras que un $13.2 \%$ en los pagos de derechos de agua potable y drenaje; los cuales suman un $63.3 \%$ (Cuadro 2). Un $25.4 \%$ de los trámites y servicios no están especificadas. El restante $11.3 \%$ está ubicado en trámites de actas de Registro Civil, cultura física y deporte, y en consultas del Portal de Obligaciones de Transparencia.

\section{Cuadro 2. Gestión de pagos y derechos de servicios públicos en municipios y delegaciones}

\begin{tabular}{|c|c|c|}
\hline \multirow[b]{2}{*}{ Año } & \multicolumn{2}{|c|}{2016} \\
\hline & $\begin{array}{l}\text { Millones de } \\
\text { Unidades }\end{array}$ & $\begin{array}{c}\% \text { de los } \\
\text { Municipios }\end{array}$ \\
\hline \multicolumn{3}{|l|}{ 1. Pago de Impuesto Predial } \\
\hline No. de trámites y servicios & 102.5 & 50.1 \\
\hline \multicolumn{3}{|c|}{ 2. Pago de derechos por agua potable y drenaje } \\
\hline No. de trámites y servicios & 27.0 & 13.2 \\
\hline Subtotal & 129.5 & 63.3 \\
\hline \multicolumn{3}{|c|}{ 3. Copia certificada del acta de Registo Civil } \\
\hline No. de trámites y servicios & 8.6 & 4.2 \\
\hline \multicolumn{3}{|l|}{ 4. Cultura física y deporte } \\
\hline No. de trámites y servicios & 8.0 & 3.9 \\
\hline \multicolumn{3}{|c|}{ 5. Consulta al portal de obligaciones de transparencia } \\
\hline No. de trámites y servicios & 6.5 & 3.2 \\
\hline Subtotal & 23.1 & 11.3 \\
\hline \multicolumn{3}{|c|}{ 6. Otras consultas no especificadas } \\
\hline No. de trámites y servicios & 52.0 & 25.4 \\
\hline
\end{tabular}

Fuente: Elaboración con base en datos del INEGI, Censo Nacional de Gobiernos Municipales y Delegacionales, 2017.

La mayoría de los gobiernos municipales, de un total de 2,458 municipios, poseen una fuerte debilidad en la capacidad institucional. No obstante, el 75\% de los municipios si dispone de una oficina administrativa (Cuadro 3). El 25\% de ellos ya cuenta con dos o más oficinas. En cuanto a obra pública, el 6\% de los municipios no tienen oficinas en 
esta materia, pero, en servicios públicos no hay oficinas en el $4.3 \%$ de los municipios. Cuando se refiere al Desarrollo Integral de la Familia (DIF) el 12\% de los municipios no cuentan con oficinas.

\section{Cuadro 3. Capacidad administrativa e institucional en los municipios}

\begin{tabular}{|c|c|c|}
\hline \multirow[b]{2}{*}{ Año } & \multicolumn{2}{|c|}{2016} \\
\hline & Unidades & $\begin{array}{c}\% \text { de los } \\
\text { Municipios }\end{array}$ \\
\hline \multicolumn{3}{|c|}{ 1. Oficina con funciones de tesorería o finanzas } \\
\hline Con una oficina administrativa & 1,843 & 75.0 \\
\hline $\begin{array}{l}\text { Con dos o más oficinas } \\
\text { administrativas }\end{array}$ & 615 & 25.0 \\
\hline Total & 2,458 & 100.0 \\
\hline \multicolumn{3}{|l|}{ 2. Oficinas de obras públicas } \\
\hline Con una oficina administrativa & 2,310 & 94.0 \\
\hline No cuenta con oficina & 148 & 6.0 \\
\hline Total & 2,458 & 100.0 \\
\hline \multicolumn{3}{|l|}{ 3. Oficinas de servicios públicos } \\
\hline Con una oficina administrativa & 2,352 & 95.7 \\
\hline No cuenta con oficina & 106 & 4.3 \\
\hline Total & 2,458 & 100.0 \\
\hline \multicolumn{3}{|c|}{ 4. Desarrollo integral de la familia } \\
\hline Con una oficina administrativa & 2,158 & 87.8 \\
\hline No cuenta con oficina & 300 & 12.2 \\
\hline Total & 2,458 & 100.0 \\
\hline
\end{tabular}

Fuentes: Elaboración con base en datos del INEGI, Censo Nacional de Gobiernos Municipales y Delegacionales, 2017.

Entre los datos del INEGI, existe un $84.8 \%$ de los municipios cuentan con oficinas de gestión catastral, algunos hacen simultáneamente la gestión de catastro y cobro de predial, y otros sólo el cobro del impuesto predial (Cuadro 4). En contraste, el 15.2\% restante no tiene oficinas con funciones de catastro, o bien, no dispone de información a este respecto. 


\section{Cuadro 4. Gestión de catastro y cobro del impuesto predial en municipios y delegaciones}

\begin{tabular}{|c|c|c|}
\hline \multirow[b]{2}{*}{ Oficina Catastral } & \multicolumn{2}{|c|}{2016} \\
\hline & Unidades & $\begin{array}{l}\% \text { de los } \\
\text { Municipios }\end{array}$ \\
\hline \multicolumn{3}{|l|}{ 1. Con gestión catastral } \\
\hline Oficinas municipales & 433 & 17.6 \\
\hline \multicolumn{3}{|c|}{ 2. Con gestión catastral y cobro de predial } \\
\hline Oficinas municipales & 851 & 34.6 \\
\hline \multicolumn{3}{|c|}{ 3. Solo cobro de impuesto predial } \\
\hline Oficinas municipales & 802 & 32.6 \\
\hline Subtotal & $2,086.0$ & 84.8 \\
\hline \multicolumn{3}{|l|}{ 4. Sin actividad catastral } \\
\hline Oficinas municipales & 352 & 14.3 \\
\hline \multicolumn{3}{|l|}{ 5. Sin datos especificados } \\
\hline Oficinas municipales & 20 & 0.8 \\
\hline Subtotal & 372 & 15.1 \\
\hline Total & 2,458 & 100.0 \\
\hline
\end{tabular}

Fuente: Elaboración con base en datos del INEGI, Censo Nacional de Gobiernos Municipales y Delegacionales, 2017.

En conjunto, estos datos revelan qué tan débil es la capacidad institucional en los municipios y delegaciones. Hay pocos datos actuales, respecto al marco regulatorio y los planes de desarrollo en los gobiernos locales, pero esta debilidad depende del trabajo legislativo en los congresos de los estados y de la poca capacidad administrativa en los ayuntamientos y cabildos, carentes de las acciones de evaluación y supervisión del gasto. Más aún, las haciendas locales no disponen muchas veces del personal capacitado para realizar tareas administrativas, menos de programas que busquen el desarrollo de ese personal en asuntos financieros.

Por supuesto, existen gobiernos municipales que pueden excluirse de esta situación. Pero, en general, no se asume una responsabilidad fiscal de los fondos públicos, más allá de una recaudación del impuesto 
predial y el cobro de derechos. Por consecuencia, es conveniente analizar y evaluar las posibilidades reales de una descentralización fiscal, dada la heterogeneidad de las estructuras administrativas a nivel de los gobiernos locales.

\section{Alternativas de política pública frente a las ineficiencias de la capacidad institucional}

La economía del país se encuentra en fiscal stuck, debido a que no hay opciones factibles de tributación, más allá de gravar la renta neta o el ingreso patrimonial de la clase empresarial (Retchkiman, 1979) ${ }^{19}$. Ante la necesidad de aumentar los recursos públicos y atender las demandas sociales, no puede aplazarse a un futuro lejano. Al menos, hay cinco opciones disponibles:

a) Elevar la recaudación fiscal, con una mayor base gravable y de contribuyentes;

b) Aumentar la disciplina en el gasto público corriente;

c) Aumentar la eficiencia de la administración tributaria;

d) Disminuir la evasión y la elusión fiscal; y

e) Aplicar la ley y evitar regímenes especiales y de privilegios.

Al elevarse la eficiencia recaudatoria y la liberación de recursos públicos, se podrían dirigir más recursos a proyectos y programas públicos, especialmente a la población más pobre. Una mayor eficiencia recaudatoria, lógicamente, tendrá necesariamente una mayor carga tributaria; a través de nuevos impuestos o de una mayor eficiencia del sistema recaudatorio (Martner y Aldunate, 2006: 88). En De Szentes y Backsay (2010), se afirma que la distribución de la carga fiscal se dirige cada vez más hacia las clases trabajadoras ${ }^{20}$, siendo que los impuestos reper-

19 Para Benjamín Retchkiman (1979), el problema es en la incapacidad del sistema impositivo para obtener recursos de aquellos contribuyentes con mayores ingresos y riqueza.

20 Se cree que hay un papel activo del Estado, desde un enfoque fiscal-marxista, que se intenta influir con los impuestos en la redistribución de la riqueza (De Szentes \& Bacskay, 2010). 
cuten en la asignación de recursos, con efectos sociales y económicos no deseables.

En el país, si bien ha habido estímulos fiscales para restablecer el crecimiento de la economía en su conjunto, no ha sido suficientes para que se desarrollen las economías estatales. La política fiscal ha tenido cambios discrecionales, sin una "ancla" a mediano plazo, lo que dio origen a riesgos fiscales y al deterioro de los marcos institucionales fiscales. No ha sido posible reconstituir los márgenes de maniobra fiscal, como una prioridad frente al futuro, ante la dinámica de la deuda y un crecimiento bajo de la economía (Celasun et. al., 2015).

Hay una relación entre federación y gobiernos de los estados y municipios, que presentan dificultades de coordinación fiscal en materia de ingresos y gastos. Todavía no hay una descentralización fiscal que realmente opere con eficiencia y sanas prácticas. Para la Comisión Económica para América Latina y el Caribe (CEPAL, 1998) la descentralización produce nuevos desafíos y se ha vuelto una fuente importante de desequilibrios fiscales. Asimismo, la centralización de los ingresos y la descentralización del gasto público a distintos niveles de gobierno, pese a su autonomía entre sí, está resultando contraria a elevar el bienestar (Ramírez, 2012), por ello es conveniente el rediseño de estrategias para un federalismo fiscal eficiente y transparente.

Ante las presiones de las demandas sociales y la poca oferta de los gobiernos locales, es posible no poder controlar los equilibrios presupuestales (Aguilar, 2010). Las finanzas públicas están expuestas constantemente a poseer un déficit fiscal, que aunado al desprestigio institucional (Uvalle, 2002) se ven problemas serios en la capacidad de gobernar. Lo que no necesariamente lleva a una crisis o a ser "factor de desgobierno" (Aguilar, 2010), pero no se generan las condiciones de confianza y certidumbre en la población.

Los problemas de gobernanza aparecen con una débil política fiscal, que se caracteriza por una baja recaudación tributaria, son reflejo de las ineficiencias y debilidades de la administración tributaria (Amieva-Huerta, 2002) a nivel federal y local; se identifica con una disfun- 
cionalidad en la capacidad institucional del sistema. En esto hay la coexistencia de factores, alrededor de la evasión y la elusión tributaria, como son las declaraciones fiscales complicadas, la cultura del no pago de impuestos, la corrupción, la atención preferencial a grandes contribuyentes y las aglomeraciones en las oficinas del Sistema de Administración Tributaria.

En lugar de ser un asunto de contribuir en un sistema fuerte, el problema de la organización fiscal es que su estructura no puede cambiarse de un día para otro. Los impuestos y subsidios, si bien no han servido de instrumentos, si están propiciando un crecimiento de actividades formales e informales. El aumento de la informalidad si está representando una limitación en la capacidad de la recaudación tributaria (Castillo y Hernández, 2012), no sólo porque se está convirtiendo en un fenómeno no controlable en el largo plazo, acentuado por la desigualdad social, sino porque no se generan condiciones apropiadas para el registro de esos contribuyentes.

No es posible observar la actuación de los gobiernos, como simples ejecutores de las políticas públicas, sino que hay una estructura institucional que toma decisiones y tiene interrelación con los contribuyentes, lo que genera resultados en materia de finanzas y políticas públicas (Hillman, 2009). La forma y los mecanismos que actúan en la integración de las finanzas federales, necesita entender la dinámica que hay en torno a las finanzas locales.

La promoción del desarrollo regional, sin duda, tiene relación estrecha con las decisiones de gasto en los gobiernos locales, así como de la distribución de las facultades y la responsabilidad tributaria (CEPAL, 1993; Carrera, 2006). Esto involucra un proceso de descentralización fiscal (Cepal, 1993: 8) ${ }^{21}$, que se asocia a una disfuncionalidad de la capacidad institucional del sistema. El crecimiento de las economías locales no depende de su dotación de recursos e infraestructura y de su

21 Es un proceso de transferencias de competencias y recursos desde la administración central hacia las administraciones sub-nacionales: estatales o municipales (Cepal, 1993: 8). 
participación en las transferencias federales, sino de los factores político-institucionales, que involucran el sistema fiscal en los tres órdenes de gobierno.

¿Qué tanto el proceso de recaudación-gasto público, asociado a la capacidad institucional se volvió un problema? Con esta pregunta, el problema es encontrar explicaciones de los fenómenos de baja recaudación y el deterioro de la economía. Al parecer no tiene su origen exclusivamente en las políticas públicas, sino connotaciones más estructurales, que implica cultura, leyes y el funcionamiento de las instituciones. Por supuesto, la no confianza en las instituciones y la aplicación de las leyes deriva en la percepción de "fallas directivas" (Aguilar, 2010), que incluso generan problemas de gobernabilidad, corrupción y de incapacidad de las instituciones. No es posible poner énfasis en analizar la actuación de los agentes, con base en información o su grado de racionalidad. Las instituciones públicas son estratégicas, por la interacción entre los actos de gobierno y la sociedad.

De ahí, la importancia de una teoría de las organizaciones en el ámbito fiscal, que no implique la ausencia de racionalidad y un enfoque del poder. Debe reconocerse la posibilidad de desarrollar mejores prácticas hacia la política pública (Williamson, 1981, 1989). Las relaciones de intercambio conllevan no sólo aspectos de cantidad y precios, sino de contratos, confianza, negociación y el estado de derecho. Lo cual juega un papel importante el Estado y el ejercicio de las políticas públicas. No es menor que la estructura fiscal y las instituciones sean artífices de una conducta, no apropiada, que se acepta o rechaza el cumplimiento de contratos y leyes, lo cual son condiciones que no reflejan un mejor sistema fiscal.

\section{Conclusiones}

Si bien el federalismo fiscal y la descentralización convocan a un asunto de la relación entre el gobierno federal y los gobiernos locales, también implica la existencia de un conjunto de disfuncionalidades en el sistema fiscal, que de no corregirse se crean problemas en la capacidad 
institucional y de pocos ingresos fiscales, frente a las necesidades de recursos públicos. Esta dependencia de la federación, no logra revertirse por los escasos incentivos para elevar los recursos disponibles. Sin embargo, se debe reconocer que los gobiernos locales no hacen los suficiente para que el gasto sea eficiente, ni para elevar la recaudación de ingresos propios.

En cuanto a la estructura de este trabajo, se observa que, al analizar los factores institucionales de la estructura fiscal, se detecta una falta de capacidad y disfuncionalidad institucional, se obliga a un financiamiento dependiente de las participaciones federales. Una descentralización difícil, en la práctica, debido a la alta dependencia de los recursos de la Federación. De ahí, que la descentralización viene atada a la propia centralización de recursos federales, que emana del propio sistema. Se carece de un sistema realmente descentralizado y que, en la ausencia de una recaudación efectiva en los gobiernos locales, lo hace precario y débil, con problemas de capacidad institucional y eficiencia en el gasto.

No ha sido fácil resarcir el deterioro y la mayor desigualdad entre la federación y los gobiernos locales, a través de mecanismos institucionales. Esto seguramente si bien acarrea problemas en el limitado papel de las políticas públicas, no es posible verificar en esta ocasión que los gobiernos estatales y municipales no han empleado correctamente sus políticas para fortalecer su capacidad institucional.

Hay evidencia de un cierto agotamiento del modelo de tributación y del sistema federal, frente a un régimen que no fortalece la descentralización, por el lado de la recaudación de ingresos propios. Lo que lleva a una evidente disfuncionalidad en la capacidad institucional, que tiene repercusiones en la debilidad de controles y la aplicación de la Ley. En este trabajo se analizaron los factores del sistema, que llevan a la dificultad y la no sostenibilidad de las finanzas públicas, tanto a nivel federal, como en los gobiernos locales. Se halló que hay disfuncionalidad de la capacidad institucional del sistema, frente a los riesgos, que impone menores márgenes de maniobra en la ejecución de la política fiscal. 
Hay algunos factores endógenos, inherentes a los procesos de tributación y gasto, que están asociados al mal funcionamiento de las instituciones públicas y, en muchos casos, al no cumplimiento de las leyes. Esto no es ajeno a la gestión del Estado, y de gobiernos locales, cuya política pública queda entredicha en cuanto a su capacidad institucional, pero que debe concentrarse en dar más pasos en esta dirección para que se logren mejores finanzas públicas en los tres órdenes de gobierno.

Hay que reconocer que este problema fiscal, no queda como un problema más sino alcanza a impactar en las condiciones y la capacidad institucional del sistema fiscal. Se advierte una creciente desigualdad y diferencias en los estados por el acceso de los recursos públicos. Específicamente, las evidencias de este trabajo son significativas, al haber obtenido los siguientes resultados:

- Existen distintos tipos de disfuncionalidades en los gobiernos locales, administrativa, financiera y legal, que no permite evitar la dependencia de los recursos de la federación.

- La capacidad de las instituciones del sistema tiende a debilitarse, en la medida en que no hay una normatividad federal integral (a no ser por los convenios de descentralización), en coordinación con los estados y municipios.

- Falta por estimar el impacto de una disfuncionalidad en la capacidad institucional del sistema fiscal, por no reunir las condiciones de eficiencia, en solventar una propuesta fiscal de largo plazo.

El esclarecer más, en torno a los riesgos del sistema tributario y fiscal, que no está convenientemente descentralizado, implica realizar un estudio más profundo. Se sabe que hay una estructura de incentivos regulatorios, mal diseñados e ineficientes, en torno a la capacidad institucional del sistema fiscal. Los gobiernos locales quedan a merced de los ingresos y participaciones federales. Ante el deterioro del sistema fiscal y de la capacidad institucional, los gobiernos federal y local necesitan emprender reformas institucionales y fórmulas para recaudar impuestos $\mathrm{y}$ otros ingresos, a efecto de apoyar el fortalecimiento institucional y las políticas públicas en las entidades. 


\section{Bibliografía}

Aguilar, Luis F., Gobernanza: El nuevo proceso de gobernar, México: Fundación Friedrich Naumann, México, 2010, pp. 1-70.

Alor, Angélica M. y Humberto Valenzuela, "Federalismo en el Estado Mexicano", Revista El Buzón de Pacioli, Núm. especial 74, octubre 2011, pp. 1-21. Disponible en: www.itson.mx/pacioli [Consultado mayo 15, 2019].

Amieva-Huerta, Juan, Temas selectos de las finanzas pública, Porrúa, México, 2002, pp. 118, 174 y 217.

Béjar A., Luisa, "De la centralización a la descentralización, el nuevo diseño institucional del Congreso de la Unión", Foro Internacional, vol. L, núm. 1, Colegio de México, 2010. pp. 115-145.

Cabrero, E. y A. Carrera, "Fiscal Decentralization and Institutional Constrains. Paradoxes of the Mexican Case", Documento de Trabajo, DAP-CIDE, México, 2001.

Cabrero, Enrique (coord.), Los dilemas de la modernización municipal: estudios sobre la gestión hacendaria en municipios urbanos de México, CIDE/Miguel Ángel Porrúa, México, 1996.

Cabrero M., Enrique, "Capacidades institucionales en gobiernos subnacionales de México ¿Un obstáculo para la descentralización fiscal?", Gestión y Política Pública, vol. XIII, núm. 3, segundo semestre, 2004, pp. 753-784.

Carrera, Ady P., "Descentralización fiscal y desarrollo local en México", XI Congreso Internacional del CLAD sobre la Reforma del Estado y de la Administración Pública, Ciudad de Guatemala, 7 - 10 nov. 2006, pp. 1-21.

Castillo, Gerardo y Sergio Hernández, "Retos para el fortalecimiento de los ingresos tributarios en México", Finanzas Públicas, vol. 4, núm.7, Centro de Estudios de las Finanzas Públicas, Cámara de Diputados, México, 2012, pp. 155-158. 
Celasun, Oya, Francesco Grigoli, Ms. Keiko Honjo, et. al., "Fiscal Policy in Latin America: Lessons and Legacies of the Global Financial Crisis", IMF Staff Discussion Notes, núm. 15-16, Washington D.C., abril 2015, p. 40.

Comisión Económica para América Latina y el Caribe (CEPAL), El pacto fiscal: fortaleza, debilidades y desafios, CEPAL, Santiago de Chile, 1998, p. 283.

, Descentralización fiscal: marco conceptual. Proyecto regional de descentralización fiscal, Santiago de Chile, Naciones Unidas, CEPAL, 1993, p. 84.

, Globalización y desarrollo, CEPAL, Santiago de Chile, abril 2002, p. 159.

De Szentes, H. y T. Bacskay, “Teorías fiscales. Las teorías fiscales de la teoría económica burguesa-vulgar”, 2010, pp. 169-219. Disponible en: http:/grupos.emagister.com/documento/teoria_y_politica_fiscal [Consultada el 5 de abril 2018].

Eggertsson, Thrainn, Economic Behavior and Institutions, Cambridge University Press, Cambridge UK, 1990, pp. 1-357.

García, Nubia L., "La autonomía financiera, las capacidades administrativas y el diseño institucional en la contratación de deuda pública municipal", Finanzas Públicas, Cámara de Diputados, CEFP, México, vol. 6, núm. 18, México, 2014, pp. 126-205.

Goode, Richard, Government Finance in Developing Countries, Brookings Institutions, Washington D. C., 1984, pp. 1-335.

Graham, Carol, Grindle, M., Lora, E. \& Seddon, J., Improving the Odds: Political Strategies for Institutional Reform in Latin America, Inter-American Development Bank, Washington D. C., 1999, pp. 1-45.

Hillman, Arye L., Public finance and public policy: responsibilities and limitations of government, Cambridge University Press, Cambridge UK, 2009, pp. 1-759. 
INEGI, Censo Nacional de Gobiernos Municipales y Delegacionales, México, 2017.

Knack, Stephen y Philip Keefer, "Institutions and economic performance: cross country test using alternative institutional measures", Economics and Politics, vol. 7, núm. 3, 1995, pp. 207-227.

Maguire, Steve y Cynthia Hardy, "Discourse and Deinstitutionalization: The decline of DDT", Academy of management journal, vol. 52, 2009, pp. 148-178.

Martner, Ricardo y Eduardo Aldunate, "Política fiscal y protección social”, Serie Gestión Pública Número 53, ILPES (LC/L.2485-P), CEPAL, Santiago de Chile, 2006, pp. 1-49.

Meléndez Muñoz, Iraima C., Fabio Maldonado-Veloza, "El institucionalismo como factor determinante en el proceso de gobernabilidad", Economía, vol. XXXIX, núm. 38, julio-diciembre, 2014, Venezuela, Universidad de los Andes, pp. 59-86.

Meyer, John W., y W. Richard Scott, Organizational Environments, Sage Publications, Inc., Calififornia, 1983.

Oates, Wallace, "The effects of property taxes and local public spending on property values: an empirical study of taxes capitalization and Tiebout Hypothesis" Journal of Political Economy, vol. 77, núm. 6, noviembre-diciembre, 1969, pp. 957-971.

Oates, Wallace, Fiscal Federalism, Harcourt Brace Jovanovich, New York, 1972.

Pierre-Richard, Agenor y Peter Montiel, La macroeconomía del desarrollo, Fondo de Cultura Económica, México, 2000, pp. 1-947.

Powell, Walter W. y Paul J. DiMaggio, The New Institutionalism in Organizational Analysis, University of Chicago Press, Chicago USA, 1991, pp. 232-263.

Ramírez, Eduardo, "Federalismo fiscal en México y comparaciones internacionales", en José L. Calva (coord.), Reforma fiscal integral. 
Análisis estratégico para el desarrollo, vol. 5, Consejo Nacional de Universitarios y Juan Pablos Editor, México, 2012, pp. 259-288.

Retchkiman, Benjamín, Política fiscal mexicana, Problemas del Desarrollo, vol. 6, núm. 24, noviembre 1975- enero 1976, México, UNAM, 1979, pp. 83-100.

Rodden, Jonathan A,. "Reviving Leviathan Fiscal Federalism and the Growth of Government," International Organization, Cambridge University Press, vol. 57, núm. 4, 2003, pp. 695-729.

Rodden, Jonathan A., The Political Economy of Federalism, Massachuttes Institutes Tecnology, USA, August 15, 2005.

Rodrik, Dani, "Institutions for High-Quality Growth: What they Are and How to Acquire Them", NBER Working Paper 7540, Cambridge, MA: IMF, February 2000, pp. 1-49.

Rosen, Harvey, Hacienda Pública, $5^{\mathrm{a}}$ ed., Mc Graw Hill, Madrid, España, 2002.

Scott, W. Richard y John W. Meyer, Institutional environments and organizations: Structural complexity and individualism, Sage Publications, Inc., Thousand Oaks, California USA, 1994.

Simon, Hebert, "The theories of bounded rationality, en C. McGuire \& Roy Radner (comps.), Decision and Organization, American Elsevier, Nueva York, 1972, pp. 161-176.

Simon, Hebert, Administrative Behavior, $2^{\mathrm{a}}$ ed., CR 1947, The Free Press, New York, 1961, pp. 1-361.

Sour, L. y Girón, F., "The flypaper effect in mexican local governments, 1990-2006", Documento de Trabajo DTAP, núm. 217, CIDE, México, 2008, pp. 1-21.

Uvalle, Ricardo, "Los fundamentos institucionales de la gestión pública", VII Congreso Internacional del CLAD, Centro Latinoamericano de Administración para el Desarrollo (CLAD), Lisboa, Portugal, octubre 2002, pp. 1-21. 
Weber, Max, Economía y Sociedad, Fondo de Cultura Económica, México, 2014.

, La ética protestante y el espíritu del capitalismo, Fondo de Cultura Económica, México, 2011.

Williamson, Oliver, Las instituciones económicas del capitalismo, $1^{\mathrm{a}}$. ed., Fondo de Cultura Económica, México, 1989, pp. 1-380.

, "The economics of organization: The transaction cost approach", en American Journal of Sociology, 87, The University Chicago Press, Chicago, Illinois November, 1981, pp. 548-577. 